\title{
Landmine Internal Structure Detection from Ground Penetrating Radar Images
}

\author{
Federico Lombardi and Hugh D. Griffiths \\ Department of Electronic \& Electrical Engineering \\ University College London \\ London, WC1E 6BT, United Kingdom \\ f.lombardi@ucl.ac.uk, h.griffiths@ucl.ac.uk
}

\author{
Alessio Balleri \\ Centre for Electronic Warfare, Information and Cyber \\ Cranfield University, Defence Academy of the UK \\ Shrivenham, SN6 8LA, United Kingdom \\ a.balleri@cranfield.ac.uk
}

\begin{abstract}
Reliable landmine detection is still an unresolved problem. Demining operations are complex activities because of the large variety of existing landmine types, many different possible soil and terrain conditions, and environmental circumstances. Due to its ability of detecting both metallic and non-metallic objects, ground penetrating radar (GPR) is a promising method for detecting landmines that may allow faster and safer operations. As the performance of GPR is mainly governed by the target signature, the potential of discriminating target based on the presence of internal reflections could be a valuable advantage for identification and recognition process. This study demonstrates that from a set of high resolution GPR slices the internal design of the landmine can be properly imaged and characterised, confirming the applicability of the methodology and the validity of such an approach.
\end{abstract}

Keywords-Ground Penetrating Radar; Landmine detection; Target characterisation; Target imaging.

\section{INTRODUCTION}

Landmine contamination represents one of the most unacceptable threats posed to humanity. These devices have been spread over vast regions in an uncontrolled manner throughout the last four decades, including recent conflicts. The threat they pose is access denial to substantial areas of the world, bringing income loss, poverty and generally significant socio-economic hardship [1]. Global deaths and injuries from landmines have hit a ten-year high, with the latest available figures from 2015 showing a $75 \%$ increase on the previous year. Reported casualties describe that the majority of the victims are civilians, with almost a half represented by children [2].

Among all techniques that are currently under development, Ground Penetrating Radar (GPR) provides a unique detection capability to achieve operationally useful performance [3] [4]. Widely accepted as a geophysical sensing tool, it uses microwave radiations to detect electromagnetic changes throughout the subsurface, and is thus capable of detecting both metallic and non-metallic objects [5].

Considering that the majority of current landmines are moulded from plastic materials, and any metallic content is minimal or absent, this capability represents a notable advantage compared to the traditional metal detection methodology [6] [7]. In addition, it could provide high resolution 3D images of the surveyed area, allowing a deeper insight of any detected anomalies [8][9].

On the other hand, GPR still presents some technological limitations, mainly a prohibitive false alarm rate, due to misleading detections generated from objects other than the mine, such as roots, rocks and hollows, as well as other battlefield debris [10][11]. The GPR signature of such objects may be comparable to that of a landmine, and this complicates the discrimination process and slows down clearance operations [12].

As demining operations are currently very slow [13], there is a pressing requirement to develop solutions that can offer significantly higher discrimination performance [14]. Target knowledge and characterisation are therefore fundamentals to discriminate between landmines and clutter targets, whether man-made or natural [15][16].

A common characteristic that can be identified for landmines and generally man-made objects is the presence, internally, of a number of components that allow the device to work [17] [18]. These assemblies, in particular when the plastic content is high, will affect the radar signature, as they act as an additional scattering contribution. This feature does not occur in natural objects, which are commonly solid dielectric items [19].

This perspective of landmine detection represents a notable improvement in the development of GPR, considering that conventionally the technology is employed as a confirmation sensor only and not as a prime search capability.

The object of this research activity is to demonstrate the capability of GPR to provide information on the internal structure of a target and the possibility of characterising the internal structure from a set of GPR images. This potential could notably increase the level of information that can be extracted from a GPR survey.

The assessment of the achievable imaging capabilities has been carried out employing a representative inert landmine complete of all its components and a random shaped rock, representing a natural clutter object, in controlled conditions. In addition, results are validated through a comparison with the correspondent images collected from an accurately moulded landmine surrogate. The produced results, presented through a 
set of GPR time slices, demonstrate the GPR ability of recording the reflections generated from the target internal assemblies, underlining the potential of the methodology.

\section{EXPERIMENTAL CAMPAIGN DESCRIPTION}

The images presented in this paper have been acquired in a test facility available at IDS - Ingegneria dei Sistemi S.p.A, a test lane including several adjacent bays filled with different soil materials. Together with the experimental site access, to accurately and precisely scan the area, a mechanical scanner straddling the lane was provided and employed. The measurement set-up is shown in Fig. 1a and Fig. 1b.

(a)

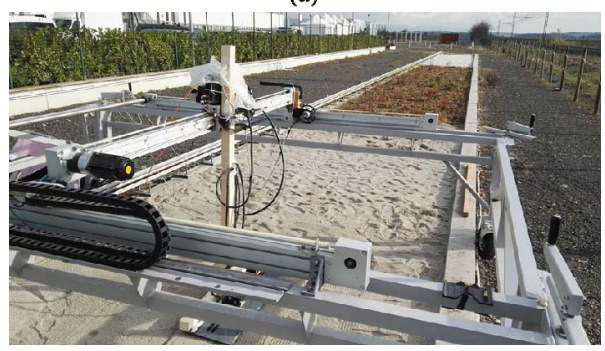

(b)

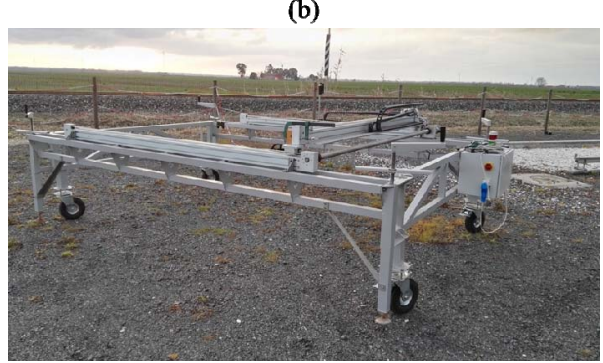

Fig. 1. Experimental test site. (a) View of the test lane. (b) Mechanical scanner employed for the measurements.

The GPR equipment employed, pictured in Fig. 2, was an IDS Aladdin radar provided by IDS Georadar srl, a shielded ground coupled dipole antenna, spaced at $6 \mathrm{~cm}$, with a central frequency and bandwidth of $2 \mathrm{GHz}$. The antenna was placed over a PVC sledge attached to the scanner. The platform has been moved along a series of parallel profiles spaced at $1 \mathrm{~cm}$, controlled by the scanner stepper motor, with a distance between inline samples of $0.4 \mathrm{~cm}$, controlled by an odometric wheel. The antennas were oriented parallel to the survey direction (vertical polarisation or TM mode).

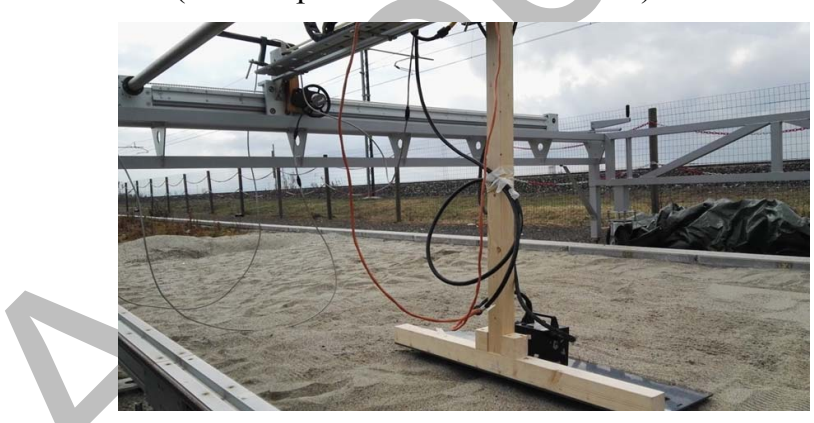

Fig. 3. Assembled GPR platform, including the odometric wheel, the mounting structure and the GPR unit.
Acquisition and equipment details are provided in Table I.

TABLE I. TRIALS DESCRIPTION

\begin{tabular}{|l|l|}
\hline \multicolumn{2}{|c|}{ Acquisition parameters } \\
\hline \multicolumn{1}{|c|}{ Parameter } & \multicolumn{1}{c|}{ Value } \\
\hline Central frequency / Bandwidth & $2 \mathrm{GHz} / 2 \mathrm{GHz}$ \\
\hline Time sampling & $0.0117 \mathrm{~ns}$ \\
\hline Inline sampling & $0.4 \mathrm{~cm}$ \\
\hline Crossline sampling & $1 \mathrm{~cm}$ \\
\hline Antenna height & $3 \mathrm{~cm}$ \\
\hline Survey area & $1 \mathrm{sqm}$ \\
\hline
\end{tabular}

Due to the environmental humidity, the sand was not completely dry, providing a propagation velocity of $10 \mathrm{~cm} / \mathrm{ns}$, a consequential dielectric value of 9 and a central wavelength of $5 \mathrm{~cm}$.

For the experimentation, a VS-50 anti-personnel landmine complete with all its external and internal components was used. The landmine was filled with a high explosive simulant commonly used to train the UK Ammunition Technical Officers. A picture of the landmine and its disassembled components is provided in Fig. 1a and Fig. 1b, respectively.

(a)

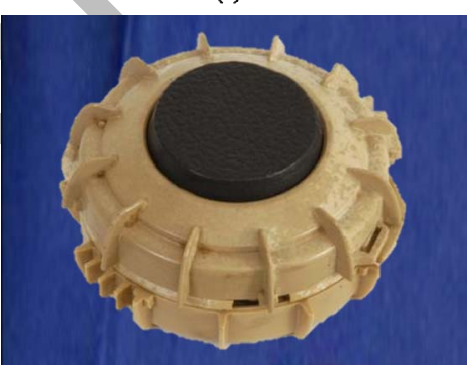

(b)

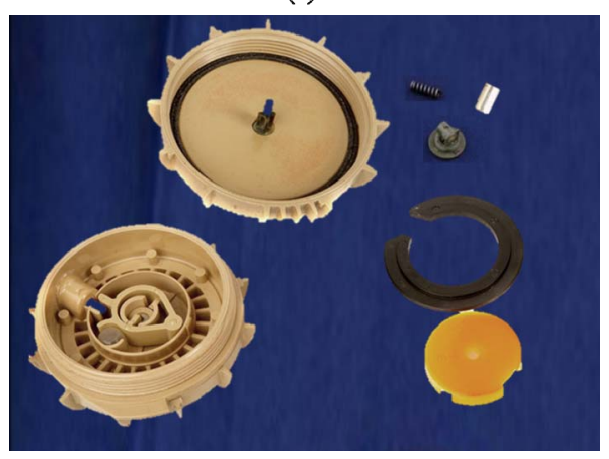

Fig. 2. VS-50 landmine details. (a) External view. (b) Disassembled components

The mine consists of a circular plastic body with vertical ribs moulded into the circumference. The mine comprises essentially three sections: (1) a main body containing the explosive charge, (2) a section carrying the fuze and the arming mechanism, and (3) the top neoprene pressure pad.

The majority of VS-50s are minimal metal mines, with a ribbed, waterproof and blast resistant plastic case. The mine incorporates an anti-shock feature which will reduce the 
effectiveness of landmine countermeasure techniques such as fuel/air explosives and explosive line charges [20]. Details of the target are provided in Table II.

TABLE II. TARGET DESCRIPTION

\begin{tabular}{|l|l|}
\hline \multicolumn{2}{|c|}{ VS-50 landmine } \\
\hline \multicolumn{1}{|c|}{ Parameter } & \multicolumn{1}{c|}{ Value } \\
\hline Diameter & $90 \mathrm{~mm}$ \\
\hline Height & $45 \mathrm{~mm}$ \\
\hline Weight & $175 \mathrm{~g}$ \\
\hline Explosive weight & $42 \mathrm{~g}$ \\
\hline Metal content & Minimum / Absent \\
\hline
\end{tabular}

As can be noticed in Fig. 3b, the middle section includes an air pressure delay mechanism, composed of an anti-shock bladder that blocks the detonation when the force on the pressure pad is of insufficient duration.

From a radar detection perspective, the presence of such assemblies is beneficial as this affects to some degree their strength and pattern of their signature. For the target under investigation, the air layer is expected to generate an additional scattering. The goal of the experimental measurements is to detect and recognise these parts, to be able to classify whether the radar anomaly can be considered as a possible buried threat.

As the final aim is to discriminate between a landmine and a clutter objects, an irregular rock (Fig. 4a) was scanned for comparison purpose. Further on, to validate the results, i.e. to confirm that the additional reflections are effectively generated by the internal assemblies of the landmine, the corresponding simulant mine provided by Fenix Insight was also characterised (Fig. 4b).
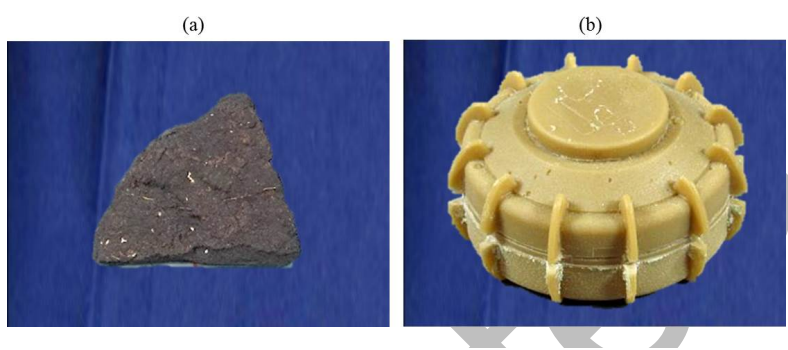

Fig. 5. Comparative targets. (a) Arbitrary shaped rock. (b) Landmine surrogate.

The surrogate is moulded from the actual mines in a resilient epoxy resin, accurately resembling in appearance the real target but without the internal assemblies. What is expected from the surrogate is an ensemble of radar images in all respects consistent with the real inert objects and characterised by a homogeneous behaviour, with no multiple and/or internal reflections.

Results are shown in terms of a set of time slices, essentially a series of radar C-scans of the volume plotting the amplitudes of the recorded data at a given time instant [21]. This allows an easy examination of the target contributions throughout its volume. Except for a time calibration to correct for jitter effects, a linear filtering to remove out-of-band noise and a spatial window, no other processing steps were applied to the data.

\section{RESULTS}

GPR slices for the representative VS-50 landmine are provided in Fig. 5. Amplitude is displayed in a blue-white-red colourmap and normalised to the range [0-1] with respect to global maximum value. As a time scale can be uniquely defined for a propagation in a homogeneous medium only, the time label for each frame has not been included.
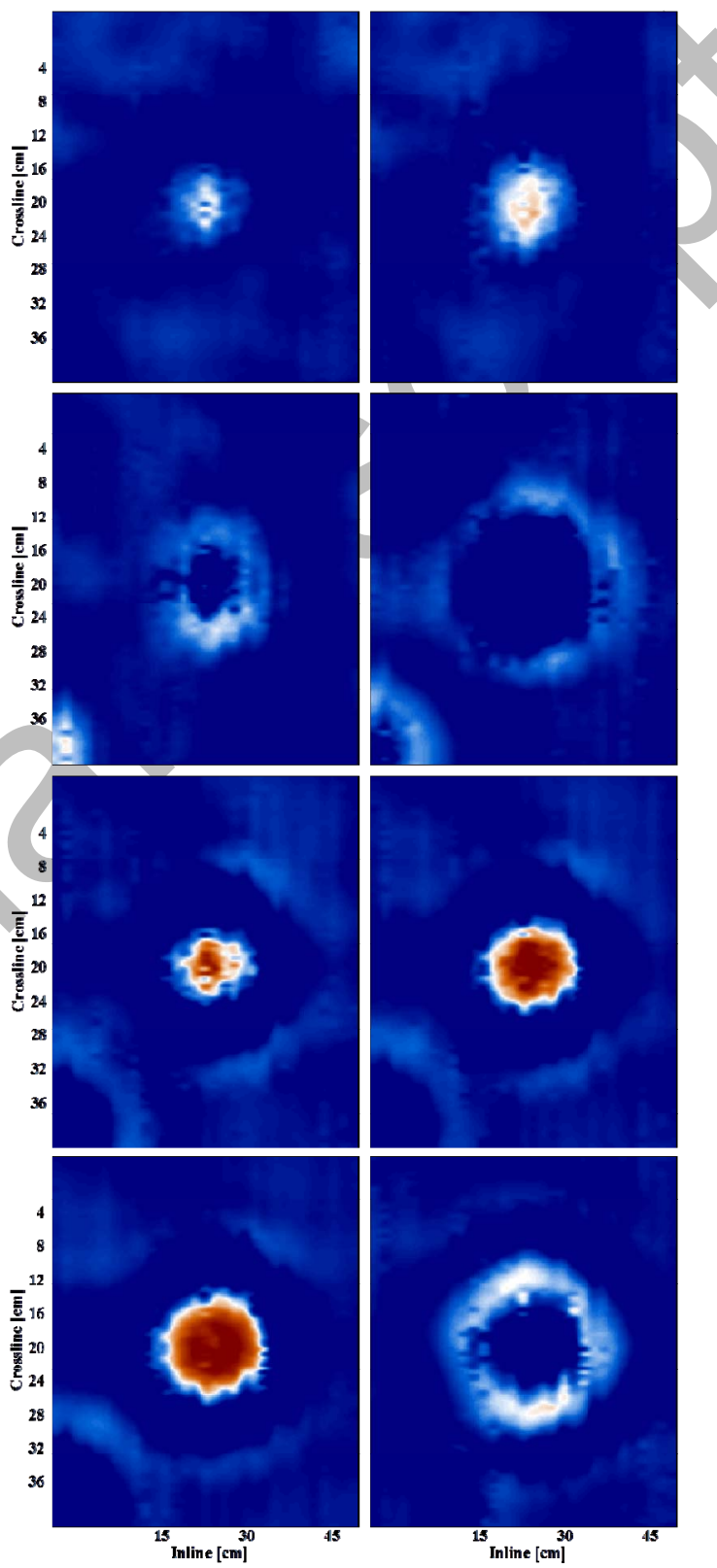

Fig. 4. Inert VS-50 landmine time slices. Order from left to right, top to bottom

A clear indication of the presence of some internal components is evident just from a preliminary analysis of the extracted slices. From a visual perspective, the reflection disappears for a couple of slices, and then the contribution from the target emerges again. What can be identified is the presence of a highly reflective layer occurring internally after the top of the landmine. 
The activator plate is clearly identifiable with the early reflections, as well as the bottom of the landmine as the last collected reflections.

A particularly valuable feature is the detection of an additional internal highly reflective area, with a slightly larger extension and higher amplitude compared to the activator pad contribution. As this layer lies within the target volume, it can be considered as an internal contribution. Recalling the landmine design, its position and geometrical properties can be plainly linked to the blast resistant component. This conclusion arises from two main considerations: (1) the intensity of the reflections is visibly higher than those generated by the contrast between the neoprene pressure pad and the surrounding soil (suggesting a substantial material difference), and (2) the area described is relatively regular and the amplitudes distribution can be regarded as homogeneous.

As a countercheck of the supposed features, the correspondent slices collected from the VS-50 surrogate are presented in Fig. 6.
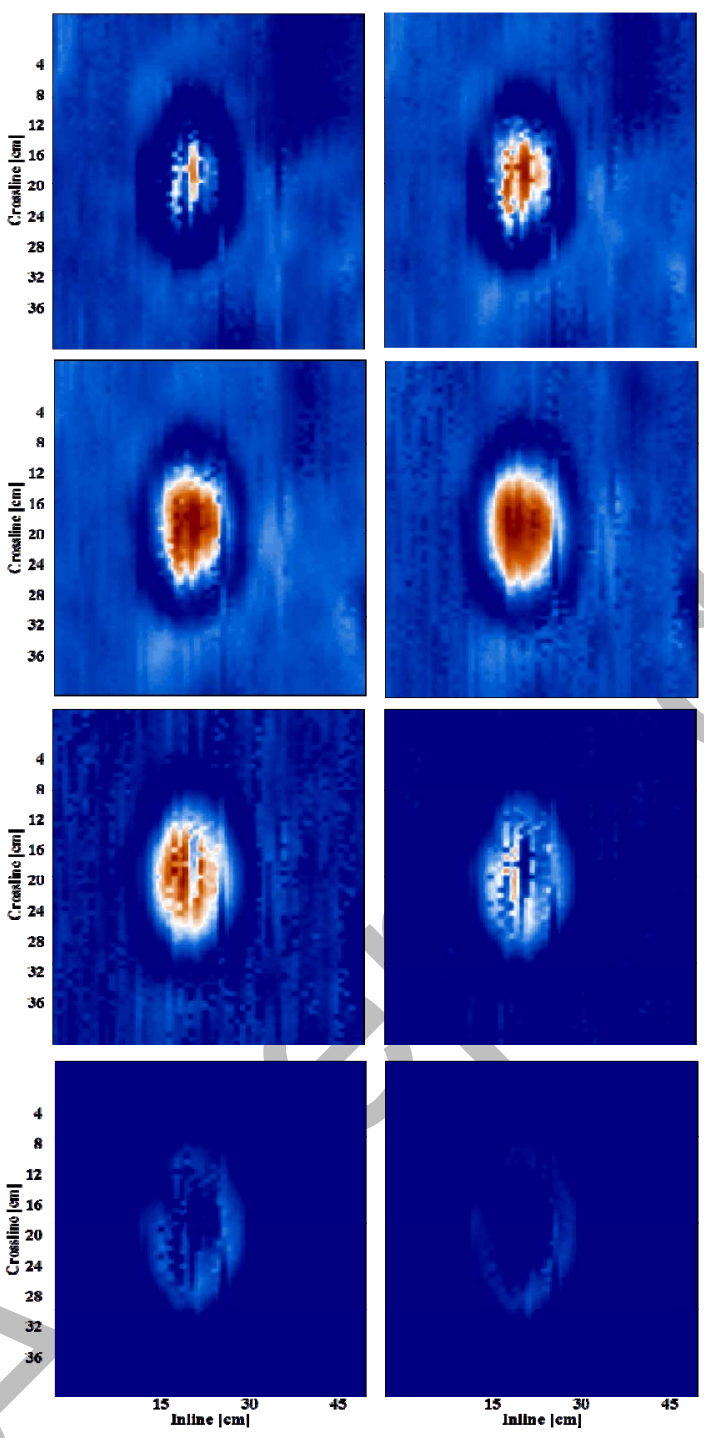

Fig. 6. Surrogate VS-50 time slices. Order from left to right, top to bottom.
The surrogate is a solid target with a representative metal inclusion; therefore a preliminary consideration is that in comparison with an air gap, a small metal inclusion has a very weak effect on the target response. Therefore, the presence of an air gap notably facilitates the detection of buried plastic cased landmines with GPR.

Examining the extracted scans, a correspondence between the surrogate and its inert equivalent cannot be found considering the target extension, as the target appears in a fewer number of slices. This is due to the neoprene pad which is a material with a velocity which is approximately $60 \%$ slower than the surrogate resin. As a figure of merit of the achieved quality of the acquisition, considering a dielectric of 3 for the surrogate, the range difference between slices is approximately $1 \mathrm{~cm}$. If one assumes six as the number of slices including the entire target, this gives an estimated height very close to the actual one $(5 \mathrm{~cm})$.

As with the previous considerations, the target exhibits a homogeneous behaviour throughout its volume, without any evidence of contributions from internal reflections.

The joint interpretation validates the hypothesis made on the nature of the reflections previously highlighted, as the only differences between the two employed targets lies in the presence of the air gap in the upper part of the representative inert VS-50.

To confirm the efficacy of the technique and to further demonstrate the discriminant nature of this feature, the arbitrary stone shown in Fig. 4a has been analysed. The target has been chosen as it represents a common source of GPR deception, and especially because it limits the advantages of a joint MD/GPR multisensor platform. Examining other possible sources of clutter, metallic debris such as bullets, crushed cans and unexploded ordnance are easily detected with the MD and moreover they will not produce meaningful GPR results. Even if they possibly may include a structure, the incident energy will be completely reflected back to the receiver. Instead, among all the possible dielectric sources of misleading detections, rocks and stones are predominant over roots and animal burrows, and are easy to evaluate. Plastic debris can be associated with the described landmine surrogate.

The slices extracted from the illustrative natural clutter object are presented in Fig. 7.

Two main considerations can be pointed out. First, the ensemble of extracted slices does not present any significant variations or alteration, suggesting the solid nature of the target. This suggestion arises also from the gradual decrease in the reflectivity area of the target, which follow the hyperbolic behaviour.

Secondly, the shape of the target is different from the previous case, underlining the advantage of having a wide band system and a high resolution capability. However, the shape cannot be considered as a robust feature, as it is dependent on the relative geometry between the buried target and the surveying equipment. 


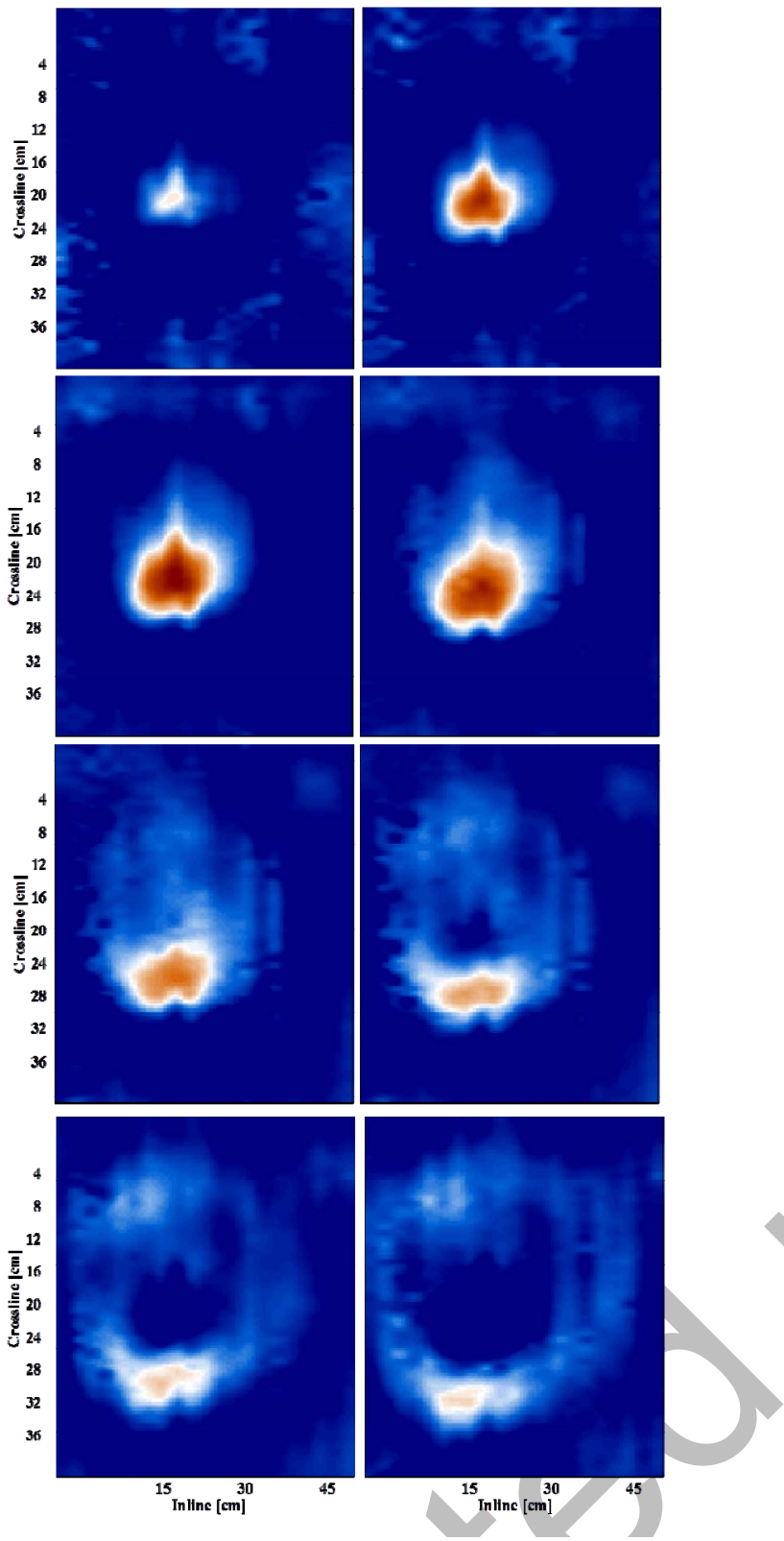

Fig. 7. Arbitrary rock time slices. Order from left to right, top to bottom.

\section{CONCLUSIONS}

The research has provided a demonstration of the ability of GPR to detect and delineate the scattering contribution generated by the presence of internal assemblies within a target.

As landmines are mostly made of plastic, with minimal metal content, and their design is complex, the challenge is (1) to be able to image the internal details, and (2) to image them with a high enough quality so that it could be beneficial for identification and recognition. Internal assemblies, in particular when the plastic content is high, will affect the radar signature, as they act as an additional scattering contribution, hardly present in natural objects. Therefore, they could represent a clear indication of buried landmines or man-made threats in general.
The experimental campaign was carried out using a wide band GPR equipment, to achieve a sufficient resolution, fundamental feature for the research scope.

The target was an inert VS-50 anti-personnel landmine, device which includes, below the neoprene activator pad, a circularly shaped series of air gaps covering the entire landmine extension.

The high resolution GPR slices that were collected showed that the internal design of the landmine can be properly imaged and characterised, confirming the applicability of the methodology. The internal assemblies of the landmine under test were identified with a high degree of confidence, even from raw radar data. In particular, a high reflectivity area below the first occurrence of the target was identified and, due to the actual landmine design, associated to the air stratum. Obviously, the dimension of each air hole is not sufficient for the equipment to be able to individually contour the contributions, but valuable information from the GPR images can be gathered.

To confirm the hypothesis, an accurately moulded surrogate, essentially a solid replica of the landmine, was analysed to properly compare the two responses, and the results reinforced the interpretation of the cause of the additional scattering plane occurring beneath the inert landmine.

Finally, as the principal scope is to reduce the false alarm rate of the technology, a series of time slices have been extracted and examined from an arbitrary stone, considered as emblematic of the family of natural clutter objects. In this case, a homogeneous pattern has been identified, oppositely to the landmine results.

In conclusion, GPR has proven to be an effective technique for imaging the possible presence of multiple internal scattering contributions, given a suitable bandwidth. This concept is fundamental, as the detection is a matter of resolution. Equally notable is that the achieved precision, even from raw data, can be a significant advantage for target identification algorithms based on image matching.

Having understood the significance of the investigated parameters, several consequent developments could be highlighted.

First of all, a single landmine model has been examined, therefore the strategy could gain a further validation employing devices characterised by different internal design. If one considers that the internal design can uniquely define a particular family of landmines, the gap between detection and recognition, and potentially identification, can be filled without expensive computational effort..

Another valuable analysis is to check whether a change in the relative geometry could impact the performance of the methodology. This is not expected for target with a regular design, but different features may emerge from inclined targets.

Finally, to assess the robustness of the recognition strategy, effects of less cooperative soil should be evaluated, as the capability of accurately outlining the internal characteristics of a target mostly relies on the achievable resolution. 


\section{ACKNOWLEDGMENT}

The authors thank the Find A Better Way charity for their support of this research under the DETERMINE programme (grant number 2015/001D). We also thank the Defence Academy Ammunition Hall for providing the landmines used for the experiments and IDS - Ingegneria dei Sistemi S.p.A. for the access to their test site. We are also grateful to A. Sarri and A. Del Moro from IDS for their support and help in the field experiments.

\section{REFERENCES}

[1] 'International Campaign to Ban Landmines', http://www.icbl.org/

[2] 'Landmine and Cluster Munition Monitor, www.the-monitor.org/,

[3] C. Bruschini, G. Bertrand. 'A survey of research on sensor technology for landmine detection', J. Convent. Weap. Destr., 2016, 2, (1), http://commons.lib.jmu.edu/cisr-journal/vol2/iss1/3.

[4] R. Keeley: 'Understanding landmines and mine action'. Available at $\mathrm{http}: / /$ mit.edu/demining/assignments/understanding-landmines.pdf

[5] D.J. Daniels: Ground Penetrating Radar (John Wiley \& Sons, Inc., Hoboken, NJ, USA, 2005).

[6] Geneva International Centre for Humanitarian Demining, 'MINEHOUND Trials 2005 - 2006: Sumamry Report', available at http://www.gichd.org/resources/publications/

[7] K. Takahashi, H. Preetz, J. Igel, 'Soil properties and performance of landmine detection by metal detector and ground-penetrating radar-soil characterisation and its verification by a field test', J. App. Geophys., 2011, 73, (4), pp. $368-377$.

[8] M.G. Aram, M. Dehmollaian, A. Khaleghi. 'Buried Target Imaging: A Comparative Study’. Sens. Imag., 2017, 18, (1), 19.

[9] Geneva International Centre for Humanitarian Demining, 'HOPE - Final Report, available at http://www.gichd.org/resources/publications/
[10] K. Takahashi, J. Igel, H. Preetz, M. Sato, 'Influence of heterogeneous soils and clutter on the performance of ground-penetrating radar for landmine detection', IEEE Trans. Geosci. Remote Sens., 2014, 52, (6), pp. $3464-3472$.

[11] D.J. Daniels, 'Ground penetrating radar for buried landmine and IED detection'. Unexploded Ordnance Detection and Mitigation, 2009, pp. 89-111.

[12] D.W. Paglieroni, D.H. Chambers, J.E. Mast, S.W. Bond, N.R. Beer.: 'Imaging modes for ground penetrating radar and their relation to detection performance', IEEE J. Sel. Topics Appl. Earth Observ. in Remote Sens., 2015 8, (3), pp. 1132 - 1144, 2015.

[13] International Standards for Humanitarian Mine Clearance Operations, http://www.un.org/Depts/mine/Standard/chap 5.htm

[14] D.J. Daniels. 'A review of GPR for landmine detection', Sens. Imag., 2006, 7, (3), pp. 90-123.

[15] K.C. Ho, L. Carin, P.D. Gader, J.N. Wilson, 'An investigation of using the spectral characterstics from ground penetrating radar for landmine/clutter discrimination', IEEE Trans. Geosci. Remote Sens., 2008 46, (4), pp. $1177-1191$.

[16] P.A. Torrione, K.D. Morton, R. Sakaguchi, L.M. Collins, 'Histograms of oriented gradients for landmine detection in ground penetrating radar data', IEEE Trans. Geosci. Remote Sens., 2014, 52, (3), pp. 1539 1550 .

[17] D.J. Daniels, P. Curtis, O. Lockwood, 'Classification of landmines using GPR', IEEE Radar Conference, Rome, Italy, May 2008, pp. 1-6.

[18] Lombardi, F., Griffiths, H.D., Balleri, A.: 'Influence of internal structure on landmine radar signatures', 13th Europ. Radar Conf., London, United Kingdom, October 2016, pp. $161-164$.

[19] F. Lombardi, H.D. Griffiths, L. Wright, A. Balleri. 'Dependence of landmine radar signature on aspect angle', IET Radar, Sonar Nav., 2017, 11, (6), pp. 892 - 902, doi: 10.1049/iet-rsn.2016.0491.

[20] Collaborative ORDnance data repository (CORD), http://ordata.info/

[21] Ö. Yilmaz. 'Seismic data analysis', Society of exploration geophysicists, (2001). 